\title{
Ion Dynamics in Single and Multi-Cation Perovskite
}

To cite this article: Ali Sehpar Shikoh et al 2020 ECS J. Solid State Sci. Technol. 9065015

View the article online for updates and enhancements. 


\title{
Ion Dynamics in Single and Multi-Cation Perovskite
}

\author{
Ali Sehpar Shikoh, ${ }^{1}$ A. Y. Polyakov, ${ }^{1, z}$ ib N. B. Smirnov, ${ }^{1}$ I. V. Shchemerov, ${ }^{1}$ D. S. Saranin, ${ }^{2}$ \\ S. I. Didenko, ${ }^{1}$ D. V. Kuznetsov, ${ }^{3}$ A. Agresti, ${ }^{2,4}$ S. Pescetelli, ${ }^{4}$ and A. Di Carlo ${ }^{4,5, z}$ \\ ${ }^{1}$ Department of Semiconductor Electronics and Device Physics, National University of Science and Technology MISiS, \\ Moscow 119049, Leninsky Ave. 4, Russia \\ ${ }^{2}$ LASE-Laboratory for Advanced Solar Energy, National University of Science and Technology MISiS, Moscow 119049, \\ Leninsky Ave. 6, Russia \\ ${ }^{3}$ Department of Functional Nanosystems and High-Temperature Materials, National University of Science and Technology \\ MISiS, Moscow 119049, Leninsky Ave. 4, Russia \\ ${ }^{4}$ CHOSE-Centre for Hybrid and Organic Solar energy, University of Rome Tor Vergata, via del Politecnico 1, 00133 \\ Roma, Italy \\ ${ }^{5}$ CNR-ISM-Istituto di Struttura della Materia CNR, via Fosso del Cavaliere 100, 00133 Roma, Italy
}

\begin{abstract}
In organic-inorganic perovskites currently widely used to fabricate high-efficiency solar cells the electrical properties are to a large extent determined by the presence of mobile ions. These mobile ions are commonly held responsible for many undesirable features of perovskite solar cells, such as hysteretic behavior of electrical properties and degradation of parameters during operation. Hence, developing methods to study the properties of mobile ions and distinguish their contribution to electrical properties from the usual effects due to electronic states are essential for gaining control over the type and density of mobile ions. In this paper we show that comparison of deep levels transient spectroscopy (DLTS) measurements performed in the normal and reverse biasing/pulsing sequences provides a useful means of discriminating between the contributions of electronic traps usual for all semiconductors and the mobile ions very important in perovskites. To simplify things these experiments were performed on Schottky diodes rather than heterojunctions with organic-inorganic electron transport and hole transport layers. The results of experiments are presented and compared for single cation $\mathrm{MAPbI}_{3}$ and multication perovskites. In both cases the main features observed in DLTS could be attributed to mobile ions.
\end{abstract}

(C) 2020 The Electrochemical Society ("ECS”). Published on behalf of ECS by IOP Publishing Limited. [DOI: 10.1149/2162-8777/ abaaf3]

Manuscript submitted July 9, 2020; revised manuscript received July 16, 2020. Published August 6, 2020.

Supplementary material for this article is available online

Metal-Organic Halide Perovskites such as $\mathrm{CH}_{3} \mathrm{NH}_{3} \mathrm{PbI}_{3}$ $\left(\mathrm{MAPbI}_{3}\right)$ have a high and strongly frequency-dependent dielectric constant, which is related to the reorientation and alignment of organic ions dipole moments. ${ }^{1-3}$ One has to also consider changes of the local arrangements of organic ions dipoles when phase transitions from tetragonal to rhombohedral structure occurs as for example in $\mathrm{MAPbI}_{3}$ near $130 \mathrm{~K}$ (and from tetragonal to cubic structure near $320 \mathrm{~K})$. At least for the low-temperature phase transition in $\mathrm{MAPbI}_{3}$ it has been reported that it gives rise to a prominent peak in dielectric constant near the phase transition temperature. ${ }^{4}$ In addition to these dipole rearrangements, one has also to consider the presence of high densities of ions with low formation energy. ${ }^{5}$ These ions are likely to be charged at whatever the quasi-Fermi level position is in perovskite layers. With applied external voltage, these ions can easily form accumulation layers near the electrodes (positive ions on the negative electrode, negative ions at the positive electrode), thus forming thin layers of space charge partially or fully screening the inner part of the perovskite film from the external electric field. This is depicted in Fig. 1 for a simple Schottky diode (SD) configuration (Fig. 1a) that we will employ in the present work, alongside with the band profile for the case without (Fig. 1b) and with (Fig. 1c) ionic accumulation at the interfaces.

In conventional semiconductors in which the band bending at the surface is created by a rectifying contact, such as a SD or p-n junction, the capacitance is due to the space charge region (SCR) near the surface where the charge of the dominant dopants, say; free electrons do not compensate donors. If the net donor concentration is $\mathrm{N}_{\mathrm{d}}$ and the band bending, close to the Schottky barrier height or the $\mathrm{p}$ - $\mathrm{n}$ junction contact voltage, is $\mathrm{V}_{\mathrm{bi}}$ the width of the space charge region $\mathrm{W}$ and the capacitance $\mathrm{C}$ are ${ }^{6}$ :

$$
W=\left[2 \varepsilon \varepsilon_{o}\left(V+V_{b i}\right) / q N_{d}\right]^{1 / 2},
$$

$$
C=\varepsilon \varepsilon_{o} S / W=S \times\left[q \varepsilon \varepsilon_{o} N_{d} /\left(2 \times\left(V+V_{b i}\right)\right]^{1 / 2},\right.
$$

where $\varepsilon_{\mathrm{o}}$ is the dielectric constant of vacuum, $\varepsilon$ is the dielectric constant of the studied material, $\mathrm{V}$ is the applied external voltage. Equations 1,2 describe the case of uniform doping and probing frequencies of AC voltage in capacitance measurements low enough that, near the edge of SCR where the donors have to capture and emit electrons alternately, the capture/emission process is fast enough to follow the applied electric field. The characteristic time for capture of electrons $\tau_{\mathrm{c}}$, and the characteristic time for electron emission from the donor, $\tau_{\mathrm{em}}$ are determined by the equation ${ }^{6}$ :

$$
\begin{gathered}
\tau_{c}^{-1}=c_{n} n, \\
\tau_{e m}{ }^{-1}=e_{n}=\sigma_{n} N_{c} v_{t h} \exp (-E d / k T),
\end{gathered}
$$

where $\mathrm{cn}=\sigma_{\mathrm{n}} \mathrm{v}_{\text {th }}$ is the capture coefficient, $\mathrm{n}$ is the concentration of free electrons, $e_{n}$ is the capture coefficient of electrons, $v$ th is the thermal velocity of electrons, $e_{n}$ is the electron emission rate, $N_{c}$ is the density of states in conduction band, Ed is the depth of donors in respect to the conduction band edge, $\mathrm{k}$ is the Boltzmann constant, $\mathrm{T}$ is the temperature. ${ }^{7}$ Equations 1, 2 are standardly used to determine the donor concentration and the barrier height from the slope and the built-in voltage $V_{b i}$ from the voltage intercept of the $1 / C^{2}$ vs voltage plots. If the distribution of donors is not uniform, it can be obtained from the differential form of Eq. $2^{6}$ :

$$
N_{d}\left(W_{d}\right)=C^{3} /\left(q \varepsilon \varepsilon_{o} S^{2}(d C / d V)\right),
$$

with the depth given by Eq. 1 .

These equations serve as the basis for concentration profiling in ordinary semiconductors. At that, the conductivity type of material is determined by the sign of $1 / C^{2}$ derivative by voltage: for $n$-type material and the negative voltage applied to the SD, capacitance decreases with voltage.

When the probing frequency becomes too high for the donors/ acceptors to follow the probing electric field in capacitance 


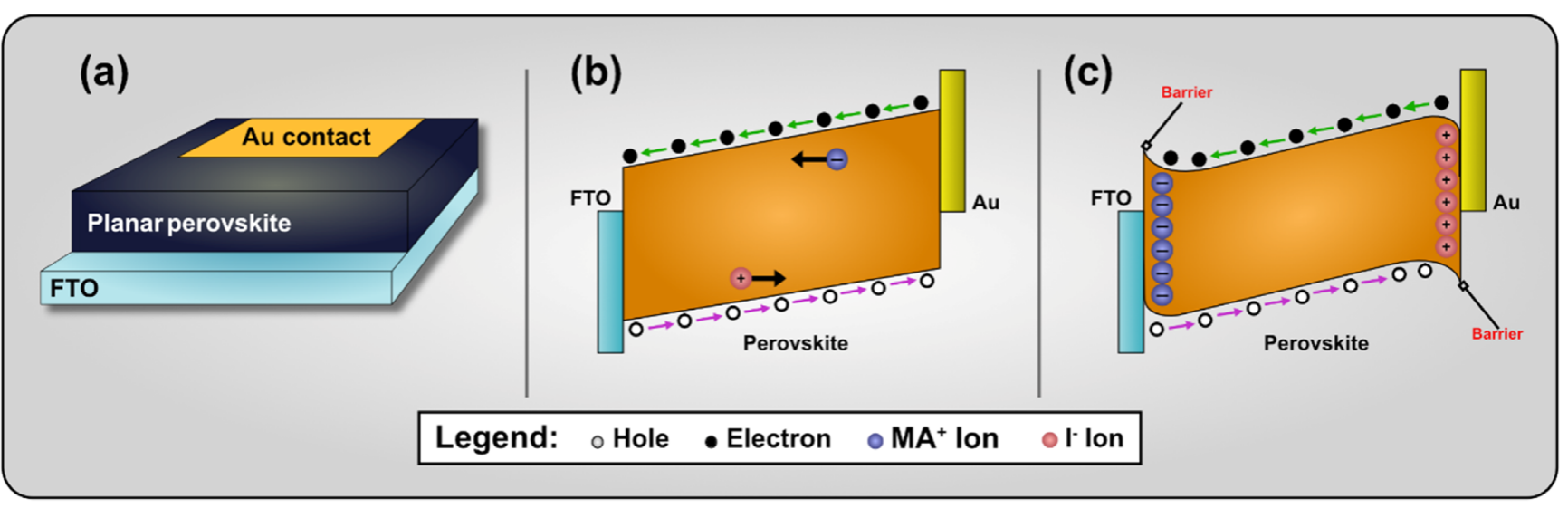

Figure 1. (a) Configuration of the fabricated $\mathrm{MaPbI}_{3}$ Schottky diode, (b) Schottky diode without ionic accumulation, (c) Schottky diode with ionic anions and cations accumulating at the interfaces of FTO/perovskite and Au/perovskite, respectively.

measurements, the whole semiconductor film behaves as a dielectric layer with geometric capacitance $\mathrm{C}=\varepsilon \varepsilon_{\mathrm{o}} \mathrm{S} / \mathrm{d}$ (where $\mathrm{d}$ is the layer thickness), and the capacitance dependence on frequency shows a step while the AC conductance $(\mathrm{G})$ shows a peak. The condition for the step in capacitance/peak in conductance to appear is ${ }^{6}$ :

$$
e_{n}=1 / \omega=1 /(2 \pi f),
$$

where $e_{n}$ is given by Eq. 4 .

If there are several donors whose levels are consequently crossed by the Fermi level as the temperature increases and the Fermi level moves deeper into the bandgap, there will be a matching number of steps/peaks. For each of these steps/ peaks, the energy of the level and the capture cross-section can be determined from Eq. 6. This is the basis of admittance spectroscopy method widely used for deep centers characterization in ordinary semiconductors. ${ }^{6}$

The situation with perovskites is different because of the presence of the two space charge layers/two large capacitances due to ions accumulation near the interfaces, as shown in Fig. 1c. Equivalent circuits of perovskite films taking into account the contribution of ions have been proposed in Ref. 2. At low frequencies, when the movement of the ions can follow the probing frequency in capacitance measurements, the contribution of the two "ionic" double layer capacitances is expected to dominate. For higher frequencies (or lower temperatures) the ions become too slow to follow the probing AC signal in capacitance measurements, and the "ionic" capacitance decreases, leaving behind the "normal" electronic capacitance attenuated by the frozen- in the electric field of the now static ionic double layer. In this regime, the equations above developed for electronic states again apply, although the actual voltage is not merely the externally applied voltage plus the band bending voltage. Also, there remains the question of which value of dielectric constant should be applied at the given temperature. However, concentration profiling by using Eq. 4 should, in principle, apply. An additional problem with PSCs having ETLs and HTLs, is accounting for the contribution of these additional layers that are partially permeable to the ions, thus contributing to their intrinsic $\left(\mathrm{MA}^{+}, \mathrm{Pb}^{+}\right.$and $\left.\mathrm{I}^{-}\right)$and extrinsic $\left(\mathrm{Li}^{+}, \mathrm{H}^{+}, \mathrm{Na}^{+}\right)$movement and eventually affecting PSC operation. ${ }^{8}$ For this reason, the interpretation of admittance spectroscopy measurements in PSCs is rather complicated. To reduce the complexity of the problem, it is possible to perform measurements on Perovskite SD consisting of a Halide perovskite semiconductor, a Schottky contact, and an Ohmic contact.

The scope of the present work is to combine different modes of DLTS (alongside capacitance and admittance measurement techniques) to PSDs in order to extract traps characteristics, such as energy levels and activation energies, of the perovskite layer and to discriminate the contribution of electronic and ionic states.

\section{Experimental}

Materials.—All solvents—dimethylformamide (DMF), dimethyl sulfoxide (DMSO) and chlorobenzene (CB) were purchased in anhydrous, ultra-pure grade from Sigma Aldrich and used as received. Schottky diodes were fabricated on SnO2:F (FTO) coated glass TEC8 from GreatCellSolar. Lead Iodide (99.99\%, trace metals basis) and lead bromide ( $99.99 \%$, trace metals basis) were purchased from TCI Chemicals; methylammonium iodide (MAI, 99.99\%), methylammonium bromide (MABr, 99.99\%), formamidinium iodide (MAI, 99.99\%), were purchased from GreatCellSolar, CsI was purchased from Sigma Aldrich.

Perovskite inks preparation.- $-\mathrm{MAPbI}_{3}$ ink was made from $\mathrm{MAI}$ and $\mathrm{PbI}_{2}$ (1:1 by molar ratio) dissolved in DMF: DMSO mixture (9:1 ratio by volume) at $1.45 \mathrm{M}$ concertation, with heating at $60{ }^{\circ} \mathrm{C}$, for $6 \mathrm{~h}$. The solution was cooled down room temperature (by allowing it to sit in the ambient environment for a duration of $5 \mathrm{~min}$ ) and was subsequently filtered through the $0.45 \mu \mathrm{m}$ polytetrafluoroethylene (PTFE) membrane, before deposition.

Triple cation perovskite precursor solution comprised of FAI (1 M), $\mathrm{PbI}_{2}(1.1 \mathrm{M}), \operatorname{MABr}(0.2 \mathrm{M}), \mathrm{PbBr}_{2}(0.2 \mathrm{M})$ and $\mathrm{CsI}(1.5 \mathrm{M} \mathrm{s})$ dissolved in the mixture of DMF:DMSO (4:1 by volume). The ink was stirred at room temperature for $30 \mathrm{~min}$ and cooled to room temperature over a 5 min duration.

Preparation of single cation perovskite SD samples.-Firstly, the patterned FTO substrates were cleaned with detergent, deionized water, acetone, and IPA in the ultrasonic bath. Subsequently, substrates were activated under UV-ozone for $30 \mathrm{~min}$. The deposition of perovskite films was done in gloveboxes with argon atmosphere $\left(<1 \mathrm{pp}\right.$, for $\mathrm{O}_{2}$ and $\left.\mathrm{H}_{2} 0\right)$. Perovskite precursor was spin-coated at 4000 RPMs $(30 \mathrm{~s})$ and $300 \mu \mathrm{l}$ of CB was dropped on the substrate on the seventh second after the start of the rotation process. Later, substrates were annealed at $100{ }^{\circ} \mathrm{C}$ for $10 \mathrm{~min}$ to form perovskite layer $450 \mathrm{~nm}$ thick. In the end, Au electrode was deposited in a thermal evaporation chamber at $2 * 10^{-6}$ Torr pressure with $1 \mathrm{~A} \mathrm{~s}^{-1}$ rate through a shadow mask to form a $0.15 \mathrm{~cm}^{2}$ active area for the pixels of the devices.

Preparation of triple cation perovskite SD samples.-Initially, FTO substrates were cleaned by an ultrasonic bath sequence composed of three steps: soap liquid dissolved in deionized water, acetone, and ethanol each phase for $10 \mathrm{~min}$. The $\mathrm{Cs}_{\mathrm{x}}\left(\mathrm{MA}_{0.17} \mathrm{FA}_{0.83}\right)_{(100-\mathrm{x})} \mathrm{Pb}\left(\mathrm{I}_{0.83} \mathrm{Br}_{0.17}\right)_{3}$ triple cation perovskite film was obtained with a one-step deposition and antisolvent method in a nitrogen-filled glovebox system. The precursor solution, composed by a mix of FAI (1 M), $\mathrm{PbI}_{2}(1.1 \mathrm{M}), \operatorname{MABr}(0.2 \mathrm{M})$, $\mathrm{PbBr}_{2}(0.2 \mathrm{M})$ and $\mathrm{CsI}(1.5 \mathrm{M})$ in the mixture of anhydrous DMF/DMSO $(3.16: 1 \mathrm{vol} / \mathrm{vol})$, was spin-coated on the clean FTO substrate in a two 
steps program at 1000 and $5000 \mathrm{rpm}$ for 10 and $30 \mathrm{~s}$ respectively. $7 \mathrm{~s}$ before the end of the second step, $200 \mu \mathrm{l}$ of chlorobenzene was poured on the spinning substrate. Immediately after spin coating, the substrates are annealed at $100{ }^{\circ} \mathrm{C}$ for $1 \mathrm{~h}$ in the nitrogen-filled glovebox. Finally, a gold electrode was deposited by the highvacuum thermal evaporation.

\section{Results and Discussion}

Capacitance measurements.-We fabricated two different sets of SDs, considering a single cation $\mathrm{MAPbI}_{3}$ perovskite and a multication CsFAMAPbIBr perovskite. The Schottky contact is obtained by the deposition of $80 \mathrm{~nm}$ of Au and using FTO as the ohmic contact (Fig. 1). ${ }^{9} \mathrm{I}-\mathrm{V}$ characteristic of fabricated SD on a singlecation film is shown in Fig. S1 of the Supporting Info (S.I.) (available online at stacks.iop.org/JSS/9/065015/mmedia).

Figure 2 shows the capacitance vs frequency plots (measured at four different temperatures) for single and multi-cation PSD samples that depict the general evolution of different capacitive processes over a range of frequencies. In the case of both types of PSDs, a capacitance plateau at high frequencies $(\sim 100 \mathrm{kHz})$ was observed, with a subsequent capacitance drop at $1000 \mathrm{kHz}$ due to the effect of resistance caused by conductive contact layers. ${ }^{10}$ The high-frequency step/plateau is the pure electronic contribution. ${ }^{11-14}$

A temperature-activated process appears at low frequencies resulting in substantial changes in capacitance. In general, lowfrequency capacitance is attributed to interfacial (electronic and ionic) charge accumulation ${ }^{12,15}$ (that affects the space charge layer and/or charge collection efficiency), since it grows to higher values when subjected to increased illumination and external field levels. In addition, the low-frequency capacitance values also depend on the type of contact layers employed.

According to Eck et al., in ideal cases, the capacitance at lower frequencies should run into saturation, thus forming a second plateau at lower frequencies, similar to the one seen in case of multi-cation $\mathrm{SD}$, (Fig. 2a). ${ }^{16}$ As seen, above room temperature, the capacitance showed a well-defined low-frequency step whose roll-off frequency increased with increased temperature. On the other hand, for single cation $\mathrm{MAPbI}_{3} \mathrm{SDs}$, we could not observe a plateau in lowfrequency $C$ - $f$ characteristics, but only an increase of capacitance with frequency (Fig. 2b). This most likely means that the frequencies used are still not low enough to accommodate the ion movement suggesting the migration energy of ions in such layers is higher than in the multi-cation perovskite films.

As the temperature decreased below room temperature, the ionic step was suppressed. C-V measurements on the high-frequency capacitance plateau showed that the films had electronic conductivity and allowed to calculate the donor concentration profiles yielding the donor densities in the range of $10^{17} \mathrm{~cm}^{-3}$ (Fig. S2).
Admittance spectra measured on these samples produced a system of steps and peaks in low-frequency capacitance and conductance, respectively, as shown in Figs. 3 and S3. The activation energy of the process in multi-cation perovskite, determined from the temperature dependence of the step (peak) in capacitance (conductance) measurements, is $0.25 \mathrm{eV}$ with a $1 / \tau \mathrm{T}^{2}$ pre-exponential factor close to $676.2 \mathrm{~s}^{-1} \mathrm{~K}^{-2}$ (see Eq. 4). Most likely, this energy is associated with the activation of ions migration, although a detailed model linking the pre-exponential factor with the ions drift/diffusion mechanism is lacking at the moment.

The capacitance dependence on temperature is also quite different between single and multi-cation perovskite. In the single cation diode, the capacitance gradually increased with temperature up to $\sim 130 \mathrm{~K}$ and then showed a sharp peak most likely related to the phase transition from tetragonal to orthorhombic phases taking place near this temperature (Fig. 4). ${ }^{17}$ The phase transition can also be seen (for frequencies $\geqslant 2 \mathrm{kHz}$ ) in the $\mathrm{G} / \omega$ vs frequency graph for $\mathrm{MAPbI}_{3}$ SD (Fig. S3b). On the contrary, the multi-cation diode presents a capacitance monotonically decreasing by lowering the temperature (Fig. 4). We can conclude that, in multi-cation perovskites, this transition is for some reason suppressed as recently suggested by. ${ }^{18}$ No low-frequency peaks in admittance related to the ions migration could be observed in the $\mathrm{MAPbI}_{3}$ single cation films in contrast to multi-cations films indicating again that the activation energies for ions migration are higher in the former case, something that is confirmed by DLTS/CDLTS measurements discussed in the next section.

DLTS/CDLTS studies.-As mentioned at the beginning, the main difference between perovskites and standard inorganic semiconductors is the presence in high concentrations of mobile ions that can (and do) form double charge layers at the interfaces with Schottky metal and ohmic contact in SD, with ETL and HTL in solar cells and in fact, at all inner interfaces inside the perovskite films, such as grain boundaries. Application of external bias causes rearrangement of these ions, driving positive and negative ions in opposite directions until they come to rest in their new positions. This re-arrangement should lead to the slow change of capacitance directly, through the change in the charge related to mobile ions, and indirectly, via the change in electric field distribution and consequent changes in the occupation of electronic states in the bandgap. These processes, of course, also reflect themselves in current transients. The task of decoupling the changes due to ionic movement and recharging of electronic states is not at all trivial and has not been solved so far. The difficulties here are related to the fact that most of $\mathrm{ab}$ initio theoretical calculations predict very high concentrations of several types of ions (on the order of $\sim 10^{18}-10^{19} \mathrm{~cm}^{-3}$ ), ${ }^{19}$ particularly those that have shallow charge transfer CT levels and should, therefore, contribute to increasing electron and hole
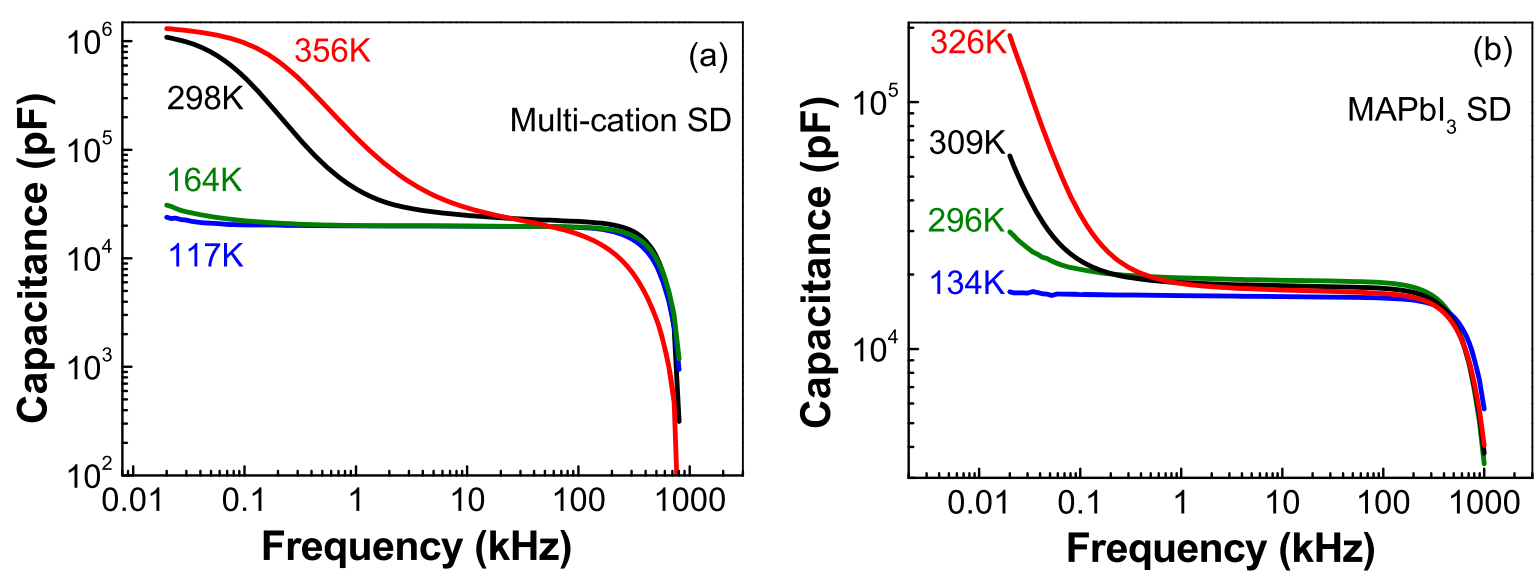

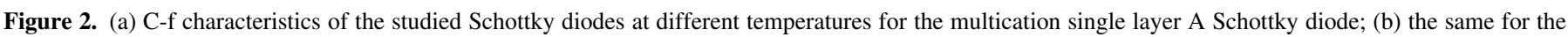
single cation $\mathrm{MAPbI}_{3}$ sample 

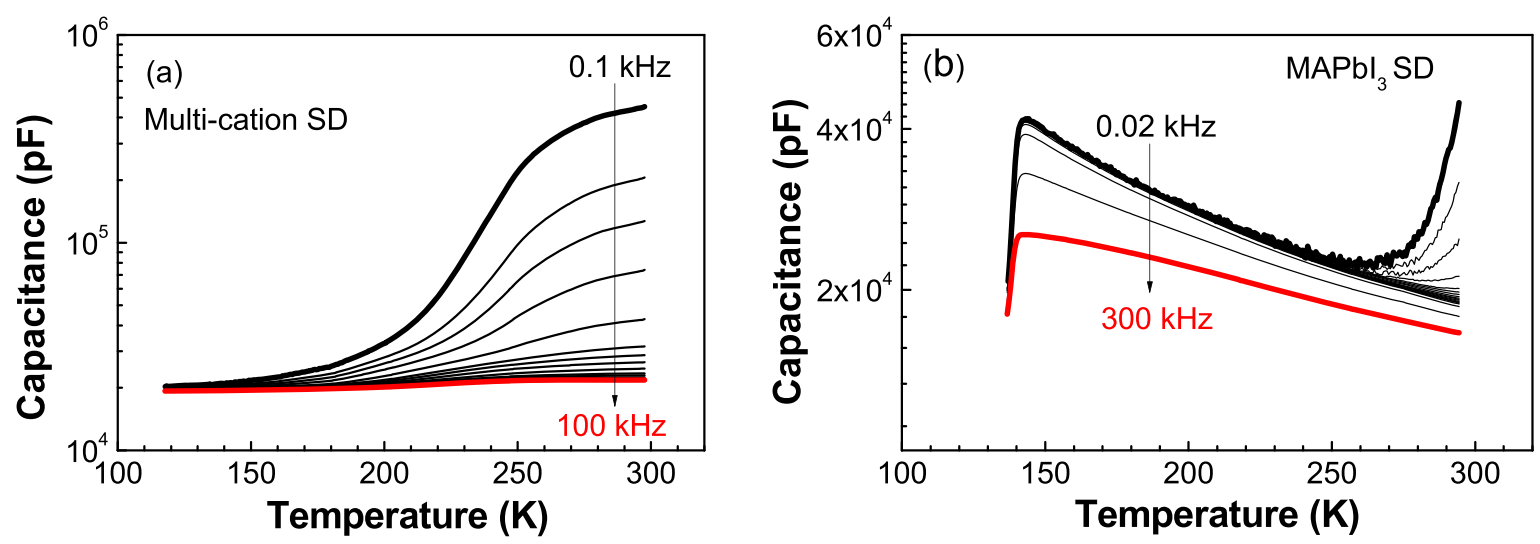

Figure 3. Capacitance vs temperature at several frequencies for (a) multi-cation and (b) single cation $\mathrm{MAPbI}_{3}$ perovskite Schottky diodes.

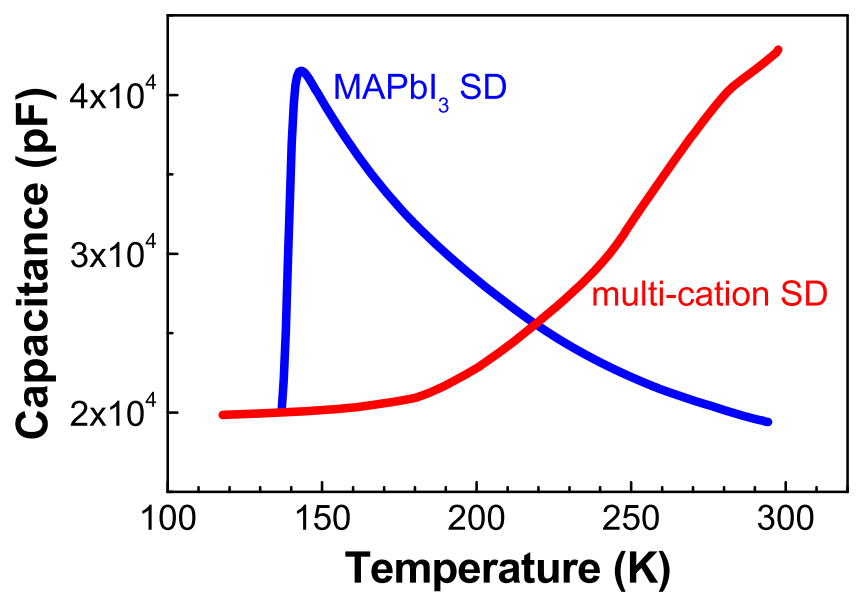

Figure 4. The temperature dependence of capacitance at $1 \mathrm{kHz}$ for the single cation and the multi-cation SDs.

concentrations. The change in the local concentration of ions should, via the requirement of sustaining local charge neutrality, promote additional changes in electron and hole concentrations supplied by the contacts, trapping of these electrons and holes should change the charge and mobility of ions. It is challenging to solve these problems self-consistently without making severe approximations. The authors are aware of only a few papers in which the problem has been quantitatively solved (see Refs. 20, 21). The primary approximation made is that the movement and trapping/detrapping of electrons and holes proceed much faster than the movement of ions and the trapping of charge carriers by mobile ions, so that at each moment the ions distributions and the distribution of free and trapped electrons and holes are "frozen" (in a way, "adiabatic" approximations). In Ref. 20 such analysis was applied to the evolution with time of open-circuit voltage of perovskite solar cells. In Ref. 22 the authors proposed to use DLTS analysis to get the migration energy, the attempt frequency in atomic hopping accounting for diffusion, and the concentration of diffusing ions assuming that they move box-to-box, from one contact to the other on the time scale much slower than the capture/emission processes due to electronic transitions in the bandgap. The concentration deduced from $\mathrm{C}-\mathrm{V}$ measurements on $\mathrm{C}-\mathrm{f}$ high-frequency plateau was used as a norm to convert the transient capacitance magnitude in such measurements into the ions concentration. Needless to say that all these approximations are difficult to justify, the weakest points, in our view, being the assumption that the electronic transitions are much faster than ionic, that the width of perovskite layer sets the distance which the ions have to travel, and that the movement of ions gives rise to exponential changes of capacitance with time.
In this work, we propose that the contributions to DLTS/CDLTS coming from mobile ions can be distinguished from electronic contributions by performing DLTS/CDLTS measurements on perovskite SD in the normal DLTS mode (i.e., when the applied voltage is pulsed from reverse to positive bias) and in reverse DLTS mode when the switching is from positive bias (low enough to allow depletion capacitance measurements) to negative bias, as represented in Fig. 5. Clearly, for electronic processes, reverse DLTS sequence in pulsing should not result in prominent capacitance transients and DLTS peaks unless the electronic capture by traps has a high energy barrier. ${ }^{7}$ On the other hand, for ionic movement, the change of the bias/pulsing sequence should result in simply oppositely charged ions accumulated near the interfaces moving in the opposite direction giving rise to similar DLTS peaks that are mirror reflections of each other.

Such measurements were performed on our perovskite SD. Figure 6a shows the normal and reverse DLTS spectra obtained for the multi-cation SD with voltage pulsing from either $-0.3 \mathrm{~V}$ to $0.3 \mathrm{~V}$ (normal DLTS) or from $0.3 \mathrm{~V}$ to $-0.3 \mathrm{~V}$ (reverse DLTS). One can clearly see the presence of two peaks A and B whose amplitudes are similar for both DLTS modes while the signs are opposite to each other, suggesting that here, in fact, we are dealing with the ionic contribution to DLTS signal. Indeed, reversing the biasing/pulsing polarity changes the direction in which the ion species accumulated near the SD and the ohmic contact move during the pulse thus varying the sign of capacitance transient (increase or decrease) after the pulse termination, similarly to the case of majority and minority traps DLTS transients in ordinary semiconductors. ${ }^{6}$ If one assumes that ions forming the double layer at the SD play the major role, one expects that, with negative bias on the Schottky electrode, positive ions will be segregated, thereby forming a depletion layer decreasing the bulk capacitance at high frequencies. Then, applying the forward bias pulse will drive the positive ions away from the Schottky metal, decreasing the double layer capacitance due to ions and increasing the overall capacitance. The capacitance relaxation is expected then to show the decay with time as for peak A in the actual spectra. For negative ions segregated at the FTO contact layer, the sign will be reversed, as for peak B. Standard DLTS analysis of the peaks temperature shift with changing the $\mathrm{t} 1$ and $\mathrm{t} 2$ time settings ${ }^{23}$ gave the activation energies for the process A as $0.24 \mathrm{eV}$ (normal DLTS) and $0.28 \mathrm{eV}$ (reverse DLTS). For process $\mathrm{B}$ respective activation energies were $0.47 \mathrm{eV}$ (normal DLTS) and $0.46 \mathrm{eV}$ (reverse DLTS). To achieve the saturation of the peaks magnitude with the pulse length one had to apply quite long pulses longer than $3 \mathrm{~s}$ (see Figs. S4a, S4b) showing the dependence of the B peak magnitude on pulse length in the case of reverse DLTS pulsing; the length of the pulses necessary to saturate the signal are similar to those reported in Ref. 21. For the single cation $\mathrm{MAPbI}_{3}$ perovskite SDs the results of DLTS measurements were qualitatively similar, but the apparent activation energies of traps $\mathrm{A}$ and $\mathrm{B}$ were higher, $0.47 \mathrm{eV}$ and $0.6 \mathrm{eV}$, as already found in the capacitance measurement of section 


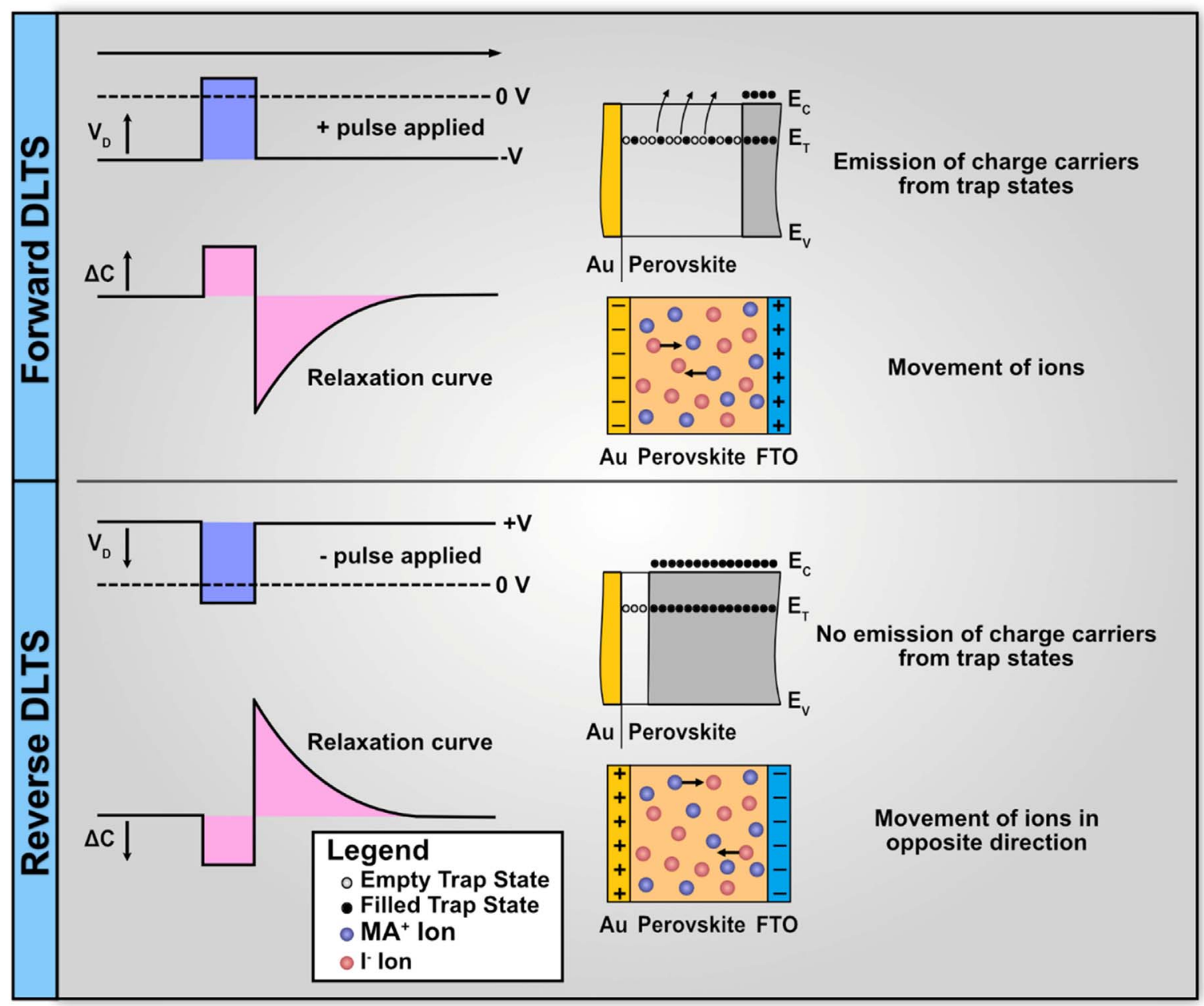

Figure 5. Schematic illustrating the key differences between conventional/forward DLTS/CDLTS and reverse DLTS/CDLTS when considering a MAPbI 3 based Schottky diode configuration with majority n-type defects.
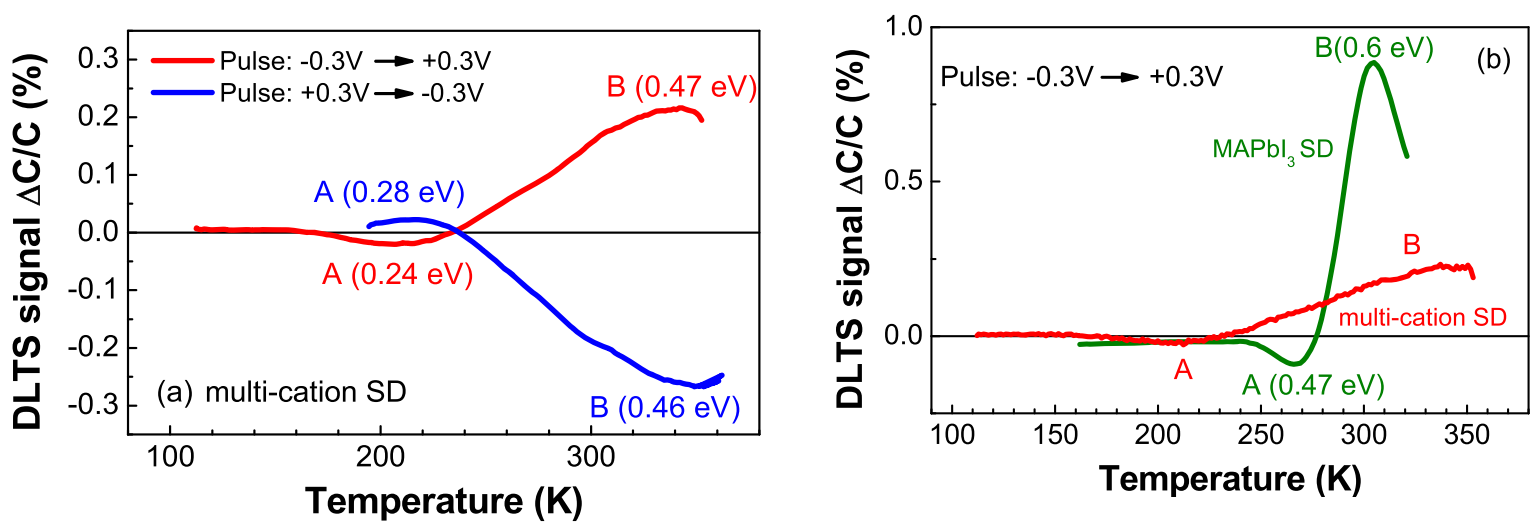

Figure 6. (a) Normal DLTS (red curve) and reverse DLTS (blue curve) spectra for multi-cation Schottky diode, $\mathrm{t}_{1} / \mathrm{t}_{2}=160 \mathrm{~ms} / 1600 \mathrm{~ms}$, pulse length $\mathrm{t}_{\mathrm{p}}=3 \mathrm{~s}$. (b) comparison of $\mathrm{MAPbI}_{3}$ and multi-cation samples spectra for normal DLTS measurements with pulsing from $-0.3 \mathrm{~V}$ to $0.3 \mathrm{~V}$ and long pulse of $3 \mathrm{~s}$.

1. Moreover, the magnitude of the peaks was about 4 times higher than for multi-cation perovskites (see Fig. 6b) comparing the spectra for normal DLTS of single and multi-cation), showing that the concentration of ionic defect states in $\mathrm{MAPbI}_{3}$ is four times higher than for the multi-cation ones.

The precise meaning of the amplitude of the DLTS peak as well as of the pre-exponential factor in the DLTS relaxation time $\tau_{\mathrm{em}}$ (Eq. 4) have yet to be determined by the relevant theory currently absent. In standard DLTS theory that describes electronic transitions,
$\mathrm{E}_{\mathrm{a}}$ is the energy level of the trap, $\tau_{\mathrm{em}}=1 / \mathrm{e}_{\mathrm{n}}$ from which the capture cross-section $\sigma_{\mathrm{n}}$ can be calculated, while the peak magnitude is proportional to concentration. It probably stands to reason to associate the activation energies deduced from "ionic" DLTS peaks with the ions migration energies, the pre-exponential factor should be in some way related to the ions transit time in the structure, whilst, for the peak magnitudes, it seems that they should be proportional to the concentration of mobile ions. Table I gives respective values of the activation energies $E_{a}$ and pre-exponential 


\begin{tabular}{|c|c|c|c|c|c|}
\hline Structure type & Method & $\mathrm{Ea}(\mathrm{eV})$ & $\sigma\left(\mathrm{cm}^{2}\right)$ & Possible origin & $\mathrm{B}$ in tau $=1 / \mathrm{B} / \mathrm{T}^{\wedge} 2 / \exp (-\mathrm{Ea} / \mathrm{kT})\left(\mathrm{s}^{-1} \mathrm{~K}^{-2}\right)$ \\
\hline \multirow[t]{4}{*}{$\mathrm{MAPbI}_{3}$} & \multirow[t]{2}{*}{ DLTS } & 0.47 & $5.5 \mathrm{e}-18$ & $\begin{array}{c}\mathrm{V}_{\mathrm{MA}^{24,25}} \\
\mathrm{~V}_{\mathrm{I}}^{25}\end{array}$ & 17710 \\
\hline & & 0.6 & $6 e-17$ & $\begin{array}{c}\mathrm{MA}^{+25} \\
\mathrm{~V}_{\mathrm{I}}^{25}\end{array}$ & 193200 \\
\hline & \multirow[t]{2}{*}{ CDLTS } & 0.45 & $3.6 \mathrm{e}-19$ & $\begin{array}{c}\mathrm{V}_{\mathrm{MA}}^{24,25} \\
\mathrm{~V}_{\mathrm{I}}^{25,26}\end{array}$ & 1159.2 \\
\hline & & 0.4 & $2.1 \mathrm{e}-19$ & $\begin{array}{l}\mathrm{V}_{\mathrm{MA}}^{25} \\
\mathrm{~V}_{\mathrm{I}}^{25,26}\end{array}$ & 676.2 \\
\hline \multirow[t]{7}{*}{ Multication } & \multirow[t]{2}{*}{ DLTS } & 0.35 & $4.1 \mathrm{e}-20$ & $\mathrm{MA}^{+27}$ & 132.02 \\
\hline & & 0.47 & $7 e-20$ & $\begin{array}{c}\mathrm{V}_{\mathrm{MA}^{24,25}} \\
\mathrm{~V}_{\mathrm{I}}^{25}\end{array}$ & 225.4 \\
\hline & CDLTS & $0.35 \mathrm{eV}$ & $6 e-20$ & $\mathrm{MA}^{+27}$ & 193.2 \\
\hline & \multirow[t]{2}{*}{ CDLTS fitting $0.3->-0.3 \mathrm{~V}$} & 0.36 & $2.7 \mathrm{e}-18$ & $\mathrm{MA}^{+27}$ & 8694 \\
\hline & & 0.18 & $4.2 \mathrm{e}-24$ & - & 0.01352 \\
\hline & \multirow[t]{2}{*}{ CDLTS fitting $-0.3 \mathrm{~V}->0.3 \mathrm{~V}$} & 0.31 & $2.85 \mathrm{E}-19$ & $\mathrm{MA}^{+27}$ & 917.7 \\
\hline & & 0.09 & $1.2 \mathrm{e}-25$ & $\begin{array}{l}\mathrm{V}_{\mathrm{I}}^{24} \\
\mathrm{I}_{\mathrm{i}}^{24}\end{array}$ & $3.864 \mathrm{E}-4$ \\
\hline
\end{tabular}

factors B in the dependence $1 / \tau_{\mathrm{em}}=\mathrm{BT}^{2} \exp \left(-\mathrm{E}_{\mathrm{a}} / \mathrm{kT}\right)$ for the studied SDs as deduced from normal/reverse DLTS. We also show the apparent capture cross-sections that would be operable were transitions of electronic type.

Similar peaks could be observed in CDLTS spectra of the studied SDs (Fig. S5). The activation energies deduced from standard CDLTS analysis are close to the activation energy of peak A in DLTS. We should point out that CDLTS measurements were performed with shorter time windows than in DLTS, $\left(t_{1} / t_{2}\right.$ of $2 \mathrm{~ms} / 40 \mathrm{~ms}$ in Fig. S5a) in order to increase the sensitivity. At such time windows settings, the CDLTS peak belonging to the B in Fig. 6 would be observed at higher temperatures where our CDLTS spectra were not taken for fear of damaging the samples. Again, for the single cation $\mathrm{MAPbI}_{3}$ sample, the peak in CDLTS (Fig. S5b) was close in energy to the A peak observed in DLTS and the energy value. As in DLTS, the energy of peak in $\mathrm{MAPbI}_{3}$ in CDLTS and the peak magnitude were higher than for the multi-cation sample (the activation energy and the apparent prefactor B are shown in Table I).

In the past, computational studies have been carried out to calculate the defect formation energies for several point defects under various conditions, including vacancies/Schottky defects $\left(\mathrm{V}_{\mathrm{MA}}, \mathrm{V}_{\mathrm{Pb}}, \mathrm{V}_{\mathrm{I}}\right)$, interstitials defects $\left(\mathrm{MA}_{\mathrm{i}}, \mathrm{Pb}_{\mathrm{i}}\right.$ and $\mathrm{I}_{\mathrm{i}}$ ) (i.e. Frenkel defects) and antisite substitutions (e.g. MAI and $\mathrm{PbI}$ ). ${ }^{25}$ Point defects comprising of vacancies and interstitials are most like defects owing to their low formation energies. ${ }^{24}$ As depicted in Table I, the activation energies close to $\sim 0.3-0.36 \mathrm{eV}$ (obtained using DLTS and CDLTS) are generally associated with electromigration of $\mathrm{MA}^{+}$ ions, which especially dominates the conductivity at high temperatures. ${ }^{27}$ This facile electromigration can be related to the material structure. ${ }^{27}$ The electromigration of MA+ ions is very fast and may take place by means of substituting $\mathrm{Pb}$ or via $\mathrm{MA}+$ interstitial sites. ${ }^{27}$ Electromigration of $\mathrm{MA}^{+}$ions is proposed as one of the primary reasons for the observed switchable photovoltaic effect. ${ }^{25}$ The activation energy of $0.46 \mathrm{eV}$ is generally associated with $V_{I}$ and $V_{M A}$ vacancies, which are the most mobile species in bulk $\mathrm{MAPbI}_{3} \cdot{ }^{10}$ Under working conditions, $\mathrm{V}_{\mathrm{I}}$ should diffuse to the negative side, whereas $V_{M A}$ should diffuse to the positive side. ${ }^{24}$

It should be noted, contrary to the box-to-box approximation, ${ }^{22}$ that the migration of ions in perovskites proceeds with different starting conditions during the capacitance/current relaxations: ions start from different positions in the film, migrate inside grain boundaries of different dimensions, undergo trapping and scattering at different numbers of grain boundaries, and experience the influence of electric field whose strength varies along the ions track.
This spread in conditions can easily result in the capacitance or current relaxation curves not being simple exponents.

Such relaxation processes occurring in disordered systems are often described by several (two in the expression below) stretched exponents of the type ${ }^{28,29}$ :

$$
I(t)=I_{o}+I_{1} \exp \left[-\left(\frac{t}{\tau_{1}}\right)^{\beta_{1}}\right]+I_{2} \exp \left[-\left(\frac{t}{\tau_{2}}\right)^{\beta_{2}}\right],
$$

where $I_{1}, I_{2}$ are constants at the fixed temperature, $\tau_{1}$ and $\tau_{2}$ are characteristic relaxation times, $\tau_{1}, \tau_{2}$ are constants below unity describing the broadening of the exponents and possibly dependent on temperature. In general, the $\beta$ factors stand for the process complexity through which the transient relaxes, whereas the two components of relaxation time $\tau_{1}$ and $\tau_{2}$ represent thermally activated mechanisms with different activation energies. ${ }^{30}$

In general, current, photocurrent, capacitance, or photo-capacitance relaxations are often described by stretched exponents in systems with various sorts of nonuniformities causing the relaxation or build-up of electrical charges following pulsed excitation. ${ }^{31}$ Physically they mean that the relaxation time during the charge build-up or decay changes in a specific range in the course of relaxation at a constant temperature that would otherwise be described by a simple exponent with a constant relaxation time. ${ }^{32,33}$ For electronic processes, stretched exponents can be due to potential fluctuations causing local variations of the quasi-Fermi level distance to the bottom of conduction band or the top of valence band, ${ }^{32,33}$ they can originate in nonuniform distribution of electric field affecting locally the actual electron capture and emission time ${ }^{29}$ the reason could be related to varying strength of the electron-phonon coupling resulting in existence of a measurable barrier for capture of electrons by deep centres. ${ }^{34}$ With ionic conductivity, the relaxation times can be caused by local changes of the activation energy of intersite hopping due to, say, the process taking place in the bulk of the grains or via the grain boundaries or in the regions with slightly different compositions. ${ }^{22}$ The pre-exponential factor variations can come from the spread in the distances that different ions have to travel in different parts of the sample and the strength of local electric field changing the relative importance of diffusion and electrically driven migration. ${ }^{22}$

The relaxation time in Eq. 7, as for simple exponents, are expected to exponentially decrease by increasing temperature with activation energies determined from numerically fitting the actual 

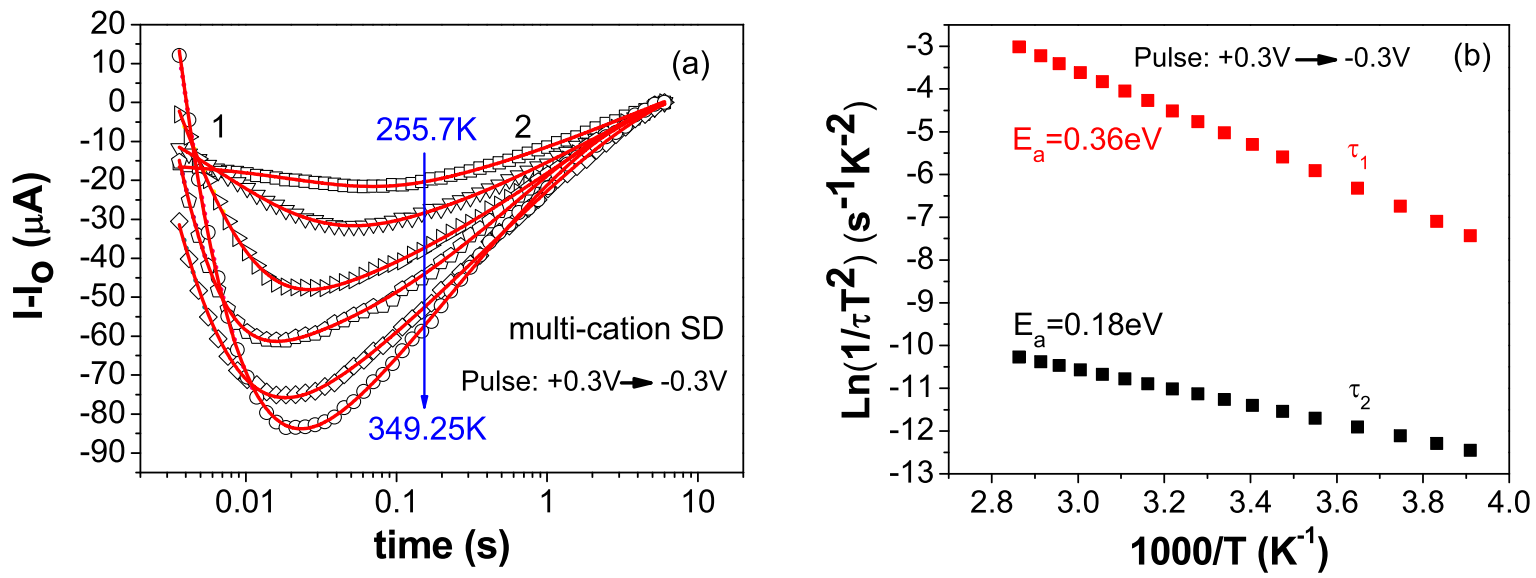

Figure 7. (a) Relaxation curves (symbols) in reverse CDLTS for several measurement temperatures fitted (continuous line) with Eq. 7, (b) Arrhenius plot of $1 /\left(\tau \mathrm{T}^{2}\right)$ for two processes in reverse CDLTS.

relaxation curves. The fitting of current relaxation curves in CDLTS was done using the procedure described in detail in Refs. 28, 29. It was shown that the relaxation curves indeed could only be adequately described by Eq. 7, but not by simple exponents with the relaxation time exponentially dependent on temperature. This form of transient/relaxation is also known as William-Watt decay and is witnessed in many physical systems, namely polymers, dielectric materials, and semiconductors. ${ }^{35}$

Figure 7 presents the results of such fitting for the multi-cation SD in the reverse CDLTS regime. The temperature dependences of the characteristic relaxation times yielded the activation energies of $0.36 \mathrm{eV}$ and $0.18 \mathrm{eV}$ for reverse CDLTS (Fig. 7b) and $0.31 \mathrm{eV}$, $0.09 \mathrm{eV}$ for normal CDLTS. Respective $\beta$ values were 0.65 and 0.4 for the former case, and 0.65 and 0.35 for the latter case. Similar A peak splitting into two processes described by stretched exponents was observed for the single cation $\mathrm{SD}$, with the first process described by the activation energy $0.45 \mathrm{eV}$ and $\beta_{1}=1$, and the second process having activation energy of $0.4 \mathrm{eV}$ and $\beta_{2}=0.75$.

\section{Conclusions}

Simplified Schottky diode structures having a perovskite active layer (sandwiched between Au and FTO contacts) were characterized using various Deep level transient spectroscopy (DLTS) and admittance spectroscopy (AS) techniques. When characterized for variation in capacitance with respect to temperature, a phase transition of MAPbI3 was clearly observed at around $130 \mathrm{~K}$. However, no such transition was observed in the case of multication perovskite samples. In comparison with single-cation samples, the ion related capacitance was more evident in multi-cation perovskite samples, suggesting comparatively lower activation energies for ion diffusion in multi-cation perovskites. In order to assess the activation energies of ions, a new forward-reverse DLTS technique was employed able to distinguish between the contribution made by electronic and ionic charged states to the DLTS signal. By comparing the forward and reverse DLTS spectrum, we showed that both multi-cation and single cation perovskite show two ionic contributions with activation energy lower in multi-cation $(\sim 0.26 \mathrm{eV}$ and $\sim 0.46 \mathrm{eV})$ with respect to single cation $(\sim 0.4 \mathrm{eV}$ and $\sim 0.6 \mathrm{eV}$ ), thus confirming admittance spectroscopy hypothesis. Moreover, the concentration of ionic defect states in $\mathrm{MAPbI}_{3}$ is four times higher than for the multi-cation perovskite. The fitting of current relaxation in CDLTS by stretched exponents shows in addition that migration of the ions occurs with different initial conditions, for example, the starting position thus the box-to-box approximation should be generally relaxed in such kind of measurements.

The proposed procedure of combined normal/reverse DLTS/ CDLTS measurements on Schottky diodes fabricated on variously prepared perovskite films allows to discriminate the contributions of the electronic and ionic signals and to determine some characteristic constants of the ionic movement, such as activation energy of movement, the value of the pre-exponential factor in the relaxation time dependence on temperature, relative magnitude of the transient capacitance or current attributable to ions in DLTS or CDLTS. Perovskite films grown with different compositions, with the addition of different ions, under different conditions, can be thus qualitatively compared. The technique can be easily extended to the analysis of processes occurring under light excitation. Of course, quantitative theory linking the measured characteristics, such as activation energy, pre-exponential factors, peak magnitudes, to physical characteristics of ions (migration energy, concentration, attempt frequency in diffusion, capture by defects), is badly needed. Nevertheless, one can try to identify the ion species responsible for the observed features based on comparisons of the migration energies calculated by theory or measured by other techniques. A good compilation of such values can be found, e.g., in Refs. 1, 21, 25, 36. We have tried to do such attribution based on these data (see Table I). However, the existing spread of values in both theory and experiment requires additional investigation on model structures. Also, the impact of the spread in the positions from which the ions in question start in DLTS-like experiments has to be better understood. It is assumed above to give rise to non-exponential behavior of mobile-ions-related capacitance and current transients, but the actual cause of the spread has yet to be explained. Some suggestions for possible causes of the spread in question have been discussed in Ref. 21. They involve the differences in $\mathrm{I}^{-}$and $\mathrm{MA}^{+}$ions migration energy in the bulk and along the grain boundaries and variation of these energies upon tetragonal to cubic phase transition at high temperature. However, detailed studies on perovskite films with different grain sizes and DLTS/CDLTS measurements in a wide range of temperatures covering the temperature of the phase transition have to be systematically performed for perovskite films of different compositions in order to better understand the nature of the processes involved. This work is currently underway in our laboratories.

\section{Acknowledgments}

The work of A.S. Shikoh at NUST MISiS was supported in part by grant № K4-2018-024 under the Program to increase Competitiveness of NUST MISiS among the World Leading Scientific and Educational centers (Program funded by the Russian Ministry of Science and Education).

\section{ORCID}

\section{A. Y. Polyakov (D) https://orcid.org/0000-0001-6898-6126}




\section{References}

1. E. Mosconi and F. De Angelis, "Mobile ions in organohalide perovskites: interplay of electronic structure and dynamics." ACS Energy Lett., 1, 182 (2016).

2. D. A. Jacobs, H. Shen, F. Pfeffer, J. Peng, T. P. White, F. J. Beck, and K. R. Catchpole, "The two faces of capacitance: new interpretations for electrical impedance measurements of perovskite solar cells and their relation to hysteresis.' J. Appl. Phys., 124, 225702 (2018)

3. A. Polyakov, N. Smirnov, I. Shchemerov, D. Saranin, T. Le, S. Didenko, D. Kuznetsov, A. Agresti, S. Pescetelli, and F. Matteocci, "Trap states in multication mesoscopic perovskite solar cells: a deep levels transient spectroscopy investigation." Appl. Phys. Lett., 113, 263501 (2018).

4. S. Govinda, B. P. Kore, M. Bokdam, P. Mahale, A. Kumar, S. Pal, B. Bhattacharyya, J. Lahnsteiner, G. Kresse, and C. Franchini, "Behavior of methylammonium dipoles in MAPbX3 $(\mathrm{X}=\mathrm{Br}$ and $\mathrm{I})$." The Journal of Physical Chemistry Letters, 8, 4113 (2017).

5. W. Ming, D. Yang, T. Li, L. Zhang, and M. H. Du, "Formation and diffusion of metal impurities in perovskite solar cell material $\mathrm{CH} 3 \mathrm{NH} 3 \mathrm{PbI} 3$ : implications on solar cell degradation and choice of electrode." Advanced Science, 5, 1700662 (2018).

6. D. K. Schroder, Semiconductor Material and Device Characterization. (John Wiley \& Sons, Hoboken, New Jersey, United States) (2015).

7. D. K. Schroder, Semiconductor Material and Device Characterization. (John Wiley \& Sons, Hoboken, New Jersey, United States) (2006).

8. Z. Li, C. Xiao, Y. Yang, S. P. Harvey, D. H. Kim, J. A. Christians, M. Yang, P. Schulz, S. U. Nanayakkara, and C.-S. Jiang, "Extrinsic ion migration in perovskite solar cells." Energy \& Environmental Science, 10, 1234 (2017).

9. A. Upadhyaya, C. M. S. Negi, A. Yadav, S. K. Gupta, and A. S. Verma, "Analysis of perovskite based Schottky photodiode." AIP Conference Proceedings, 2100(1), p. 020134 (2019).

10. O. Almora, I. Zarazua, E. Mas-Marza, I. Mora-Sero, J. Bisquert, and G. GarciaBelmonte, "Capacitive dark currents, hysteresis, and electrode polarization in lead halide perovskite solar cells." The Journal of Physical Chemistry Letters, 6, 1645 (2015).

11. F. Galatopoulos, A. Savva, I. T. Papadas, and S. A. Choulis, "The effect of hole transporting layer in charge accumulation properties of pin perovskite solar cells.' APL Mater., 5, 076102 (2017).

12. A. Guerrero, G. Garcia-Belmonte, I. Mora-Sero, J. Bisquert, Y. S. Kang, T. J. Jacobsson, J.-P. Correa-Baena, and A. Hagfeldt, "Properties of contact and bulk impedances in hybrid lead halide perovskite solar cells including inductive loop elements." The Journal of Physical Chemistry C, 120, 8023 (2016).

13. M. Salado, L. Contreras-Bernal, L. Caliò, A. Todinova, C. López-Santos, S. Ahmad, A. Borras, J. Idígoras, and J. A. Anta, "Impact of moisture on efficiency-determining electronic processes in perovskite solar cells." Journal of Materials Chemistry A, 5, 10917 (2017).

14. K. Wang, Y. Shi, L. Gao, R. Chi, K. Shi, B. Guo, L. Zhao, and T. Ma, "W (Nb) Oxbased efficient flexible perovskite solar cells: from material optimization to working principle." Nano Energy, 31, 424 (2017).

15. P. Yadav, S.-H. Turren-Cruz, D. Prochowicz, M. M. Tavakoli, K. Pandey, S. M. Zakeeruddin, M. Grätzel, A. Hagfeldt, and M. Saliba, "Elucidation of charge recombination and accumulation mechanism in mixed perovskite solar cells." The Journal of Physical Chemistry C, 122, 15149 (2018).

16. M. Eck, C. Van Pham, S. Züfle, M. Neukom, M. Sessler, D. Scheunemann, E. Erdem, S. Weber, H. Borchert, and B. Ruhstaller, "Improved efficiency of bulk heterojunction hybrid solar cells by utilizing CdSe quantum dot-graphene nanocomposites." Phys. Chem. Chem. Phys., 16, 12251 (2014)

17. K. Nakada, Y. Matsumoto, Y. Shimoi, K. Yamada, and Y. Furukawa, "Temperature-dependent evolution of raman spectra of methylammonium lead halide perovskites, CH3NH3PbX3 (X = I, Br).” Molecules, 24, 626 (2019).
18. F. Ruf, M. F. Aygüler, N. Giesbrecht, B. Rendenbach, A. Magin, P. Docampo, H. Kalt, and M. Hetterich, "Temperature-dependent studies of exciton binding energy and phase-transition suppression in (Cs, FA, MA) $\mathrm{Pb}$ (I, Br) 3 perovskites." APL Mater., 7, 031113 (2019).

19. Q. Lin, Z. Wang, H. J. Snaith, M. B. Johnston, and L. M. Herz, "Hybrid perovskites: prospects for concentrator solar cells." Advanced Science, 5, 1700792 (2018)

20. D. Walter, A. Fell, Y. Wu, T. Duong, C. Barugkin, N. Wu, T. White, and K. Weber, "Transient photovoltage in perovskite solar cells: interaction of trap-mediated recombination and migration of multiple ionic species." The Journal of Physical Chemistry C, 122, 11270 (2018)

21. M. H. Futscher, J. M. Lee, T. Wang, A. Fakharuddin, L. Schmidt-Mende, and B. Ehrler, "Quantification of ion migration in $\mathrm{CH} 3 \mathrm{NH} 3 \mathrm{PbI} 3$ perovskite solar cells by transient capacitance measurements." Materials Horizons, 6, 1497 (2019).

22. M. H. Futscher, J. M. Lee, L. McGovern, L. A. Muscarella, T. Wang, M. I. Haider, A. Fakharuddin, L. Schmidt-Mende, and B. Ehrler, "Quantification of ion migration in $\mathrm{CH} 3 \mathrm{NH} 3 \mathrm{PbI} 3$ perovskite solar cells by transient capacitance measurements." Materials Horizons, 6, 1497 (2019).

23. D. Lang, "Deep-level transient spectroscopy: a new method to characterize traps in semiconductors." J. Appl. Phys., 45, 3023 (1974).

24. J. M. Azpiroz, E. Mosconi, J. Bisquert, and F. De Angelis, "Defect migration in methylammonium lead iodide and its role in perovskite solar cell operation." Energy \& Environmental Science, 8, 2118 (2015).

25. C. Li, A. Guerrero, Y. Zhong, and S. Huettner, "Origins and mechanisms of hysteresis in organometal halide perovskites." J. Phys. Condens. Matter, 29, 193001 (2017).

26. K. Domanski, W. Tress, T. Moehl, M. Saliba, M. K. Nazeeruddin, and M. Grätzel, "Working principles of perovskite photodetectors: analyzing the interplay between photoconductivity and voltage-driven energy-level alignment." Adv. Funct. Mater. 25, 6936 (2015).

27. Y. Yuan, J. Chae, Y. Shao, Q. Wang, Z. Xiao, A. Centrone, and J. Huang, "Photovoltaic switching mechanism in lateral structure hybrid perovskite solar cells." Adv. Energy Mater., 5, 1500615 (2015).

28. A. Y. Polyakov, N. Smirnov, I. V. Shchemerov, I.-H. Lee, T. Jang, A. A. Dorofeev, N. B. Gladysheva, E. S. Kondratyev, Y. A. Turusova, and R. A. Zinovyev, "Current relaxation analysis in AlGaN/GaN high electron mobility transistors." Journal of Vacuum Science \& Technology B, Nanotechnology and Microelectronics: Materials, Processing, Measurement, and Phenomena, 35, 011207 (2017).

29. A. Polyakov, N. Smirnov, I. Shchemerov, F. Ren, and S. Pearton, "Gate-Lag in AlGaN/GaN high electron mobility transistors: a model of charge capture." ECS J. Solid State Sci. Technol., 6, S3034 (2017).

30. M. Exarchos, D. Moschou, G. Papaioannou, D. Kouvatsos, and A. Voutsas, "Performance of thin-film transistors fabricated by sequential lateral solidification crystallization techniques." Physica Status Solidi C, 5, 3634 (2008).

31. J. Lin and H. Jiang, "Relaxation of stored charge carriers in a $\mathrm{Zn} 0.3 \mathrm{Cd} 0.7 \mathrm{Se}$ mixed crystal." Physical Review B, 41, 5178 (1990).

32. H. Queisser and D. Theodorou, "Decay kinetics of persistent photoconductivity in semiconductors." Physical Review B, 33, 4027 (1986).

33. A. Dissanayake, M. Elahi, H. Jiang, and J. Lin, "Kinetics of persistent photoconductivity in $\mathrm{Al} 0.3 \mathrm{Ga} 0.7 \mathrm{As}$ and $\mathrm{Zn} 0.3 \mathrm{Cd} 0.7 \mathrm{Se}$ semiconductor alloys." Physical Review B, 45, 13996 (1992).

34. M. T. Hirsch, J. Wolk, W. Walukiewicz, and E. Haller, "Persistent photoconductivity in n-type GaN." Appl. Phys. Lett., 71, 1098 (1997).

35. J. Xia and A. Mandelis, "Broadening effects and ergodicity in deep level photothermal spectroscopy of defect states in semi-insulating GaAs: a combined temperature-, pulse-rate-, and time-domain study of defect state kinetics." J. Appl. Phys., 105, 103712 (2009).

36. B. Chen, M. Yang, S. Priya, and K. Zhu, "Origin of J-V hysteresis in perovskite solar cells." The Journal of Physical Chemistry Letters, 7, 905 (2016). 